\title{
The therapeutic use of music in a care of the elderly setting: a literature review
}

\author{
ROSALEEN KNEAFSEY \\ Third Year Undergraduate Nursing Student, Department of Nursing Studies, University of \\ Edinburgh, Adam Ferguson Building, 40 George Square, EH8 9LL \\ Accepted for publication 30 June 1997
}

\section{Summary}

- This paper reviews recent literature concerning the use of music and music therapy in health care.

- Focusing particularly on the elderly, the use of music in relation to patients with dementia and Parkinsonism is examined.

- Brief reference is also made to the use of music in pain control.

- Although in this case, literature is not specific to care of the elderly settings, the results are still relevant to gerontological nursing.

- Projects which achieved positive results in controlling pain perception could be transferable to a care of the elderly scenario, where chronic pain is often part of daily life.

Keymords: dementia, music, pain, Parkinson's disease.

\section{Introduction}

Music, an aural stimulant, has a well-documented capacity to evoke physical responses: e.g. modulation, using music, of the psycho-physiological manifestations of stress in Coronary Care Unit patients has been reported (Fitzsimmons, 1991). Music is also said to evoke psychological responses. For example, Gerdner \& Swanson (1993) suggest that individualized music can reduce agitation in confused agitated patients. It is also claimed that music can reduce situational anxiety (Evans \& Rubio, 1994), create significant mood changes (Bailey, 1985), facilitate communication (Selman, 1988) and improve mobility (Swallow, 1987).

In the last decade there has been a noticeable increase in the use of music in the health care field. Anecdotal articles describe subjectively the benefits which music can offer, and a scattering of research projects have systematically assessed the effects of music on patients. From the results and conclusions of such literature it is apparent that music for recreation, diversion and therapy has become recognized as an important therapeutic tool with scope for widespread application. The positive achievements of smallscale research programs, if possible to replicate, would be highly valuable to gerontological nursing and thus a review of the literature and evidence for such claims is warranted.

It is important to draw a distinction between the use of music in diversion and recreation and the use of music as therapy. Bruscia (1991) describes the former as 'music in therapy' where therapist and client listen to or play a musical composition. The experience of listening forms the basis for discussion of thoughts or feelings elicited by the music. The music therapist seeks to build a communicative and interactive relationship with the client, rendering meaningful the most limited responses of the individual (Pavlicevic, 1991). Improvement of functioning as the central aim of music therapy can complement the ethos of 
rehabilitation in care of the elderly settings which also seeks to maximize and achieve the highest potential level of functioning.

\section{Dementia and music therapy}

For elderly people, the potential of music as a cathartic medium is substantial. Bailey (1985) suggests that, as a patient identifies with his/her emotional state of being represented within a piece of music, it is possible to introduce other styles or instruments to alter mood. Music in synchrony with mood can act as a catalyst, releasing emotions and opening better channels of communication. Indeed, music in its multidimensional nature allows it to touch physical, psychological, spiritual and social levels of consciousness. It is this quality which allows music to reach patients during the isolation of pain or illness, brain deterioration or a decreasing ability to communicate. Music therapists say that, through intervention, meaningful communication can be re-established between patients, family and staff. This is particularly important for the dementing patient. Bright (1990) proposes that in this case music can be helpful in reorientation, rebuilding social links and raising moral. Bright (1990) also highlights grief as a common component of dementia. Music can be used as a diversion from sadness or anger often triggered by the departure of visitors. This diversion does not seek to deprive the individual of his/her right to feelings but the therapist listens and then attempts to deflect attention through the playing of music.

There have been a number of studies which have sought to discover the effects of music on demented patients. Gerdner \& Swanson (1993) investigated the effects of individualized music on five confused, agitated elderly patients with dementia of the Alzheimer's type who resided in a long-term care facility. They suggested that playing music which evoked pleasant memories would produce a soothing effect on agitated patients. Previous research by Crystal et al. (1989) and Bright (1990) had already substantiated the belief that musical perception would be retained at varying levels into the late stages of dementia. This suggested that music could be used as an alternative method of communication when the cognitive ability to receive and express language has gone.

The experimental procedure employed by Gerdner \& Swanson (1993) involved collecting baseline information on the incidence of agitated behaviours in the first week of the study. During the second week, individually selected music was played to patients for $1 \mathrm{~h}$, during which time patients' behaviour was observed and recorded in order to ascertain the immediate effects of music on behaviour. Then, immediately after listening to music, behaviour was observed and recorded to determine the residual effects of music on patient behaviour. Comparisons of baseline levels of agitation with agitation levels during music listening and post-intervention were used to calculate the percentage effectiveness of music in reducing agitation levels. The number of agitated behaviours exhibited during baseline observations was then compared with the number in the hour post-intervention for each patient.

The results showed a time lag in the effect of the music intervention in that a reduction in agitated behaviours occurred in the hours post-intervention. Gerdner \& Swanson (1993) suggest that this is because the Alzheimer's patient requires a substantial time span to process the music and, following this, for relaxation to occur. The authors also suggest that the period of time for processing and degree of response will vary as a result of certain factors, e.g. the significance of the music to the individual and the accuracy in selecting a patient's preferred songs or instruments. In this study the patient for whom music had played the most significant role had the most significant reduction in agitated behaviour. The participant for whom appreciation for music could not be ascertained exhibited the most minimal reduction in agitated behaviour. Timing of the intervention was also seen as an important factor. Implementing music before peak levels of agitation was seen as preferable in an attempt to prevent an increase in agitated behaviours. Agitation beyond peak levels may exceed the effectiveness of individualized music intervention. Gerdner \& Swanson (1993) conclude by suggesting that appreciation of music could be incorporated into the initial assessment of each patient on admission, taking into account ethnicity and religious backgrounds which could affect music preference. This approach would be inexpensive and minimally time consuming. The research findings suggest that individualized music has potential as an alternative approach to the management of the agitated elderly.

Norberg (1986) also examined the effect of music, along with touch and object presentation, on two final stage dementia sufferers. Norberg highlights how progressively worsening brain dysfunction causes symptoms such as agnosia, apraxia, aphasia and disturbances in muscular tonus which affect communication, both verbal and nonverbal. The patients' decreasing ability to communicate, although they were sometimes still aware, may lead to further psychological and social features which worsen their ability to communicate. Typically, the patients' personal territory is limited to the bed, which is also regularly invaded by carers to undertake activities such as washing and dressing. In an attempt to feel safe the patient 'withdraws' into him/herself, perhaps initiating self-stimulating behaviour. A vicious circle begins where the patient's 
ability to react to stimuli decreases, causing a deterioration in the amount of stimulation given by the carers, resulting in a further deterioration in reaction. Norberg rises to the challenge of communicating efficiently with such patients. She suggests that stimuli affecting as many sensory modalities as possible may be most effective. The aim of her study was to compare the reactions to three types of stimuli music, touch and object presentation. Two patients (A and B) were involved. Patient A communicated through eye blinking whilst patient $\mathrm{B}$ lay babbling incomprehensibly although saying the occasional recognizable word. Selfstimulation was a common pastime for patient $B$, creating withdrawal and vagueness. Music was chosen to represent what the patients had liked earlier, as described by family members. Both patients listened to the same music. Touch was given systematically as small stroking motions on face, hand, feet and back. Object presentation involved encouraging the patient to touch, smell and watch objects (soap, wool, hay) whilst the researcher talked about them. Sixteen trials lasting $90 \mathrm{~min}$ were performed whilst two independent observers observed the patients. Inter-rater reliability between observers showed that agreement of judgements of behaviours was high.

The results of Norberg's trials were measured using silent mouth movements and eye blinks for patient $A$ and incomprehensible verbal reactions and half opening of the eyes for patient B. From the results it was evident that both patients reacted differently to music than to touch and object presentation. For patient $A$ the number of blinkings during music was fewer than during touch and object presentation while the number of mouth movements was higher. For patient $B$ the registration of half-opened eyes was less during music than during touch and object presentation, whilst verbal reactions were fewer. Norberg (1986) surmises that the behaviour of patient $A$, with her eyes more open and her mouth moving more, could be understood as an orienting response. Patient B, with her eyes more closed, could be seen as relaxing. As there are no objective methods of assessing the emotional quality of the patients' reactions, the author has made the subjective assumption that both patients have responded positively to the music and were thus expected to have gained from the stimulation. Although only two patients were involved in this study, Norberg draws some tentative conclusions regarding relevance to nursing care. First, she suggests that patients in the last stages of dementia can be reached communicatively through music and secondly, that the reactions exhibited can be evaluated inexpensively. This study indicates that more research is necessary to investigate further how to make contact with such regressed patients.
In Japan, studies in the use of music therapy have looked at the effect of music on psycho-somatic diseases in senescence. Kawano (1992) states that a somato-psychological approach can be a more effective means of communication than the verbal approach. Kawano (1992) involved 14 patients with psycho-somatic diseases aged $65-81$ who listened to music of their preference for 30-60 min using a body sonic apparatus. The effects of the music were recorded by questionnaires administered to the patients. Music was found to be calming and described as good for $70 \%$ of patients at short intervals while positive effects were recorded for $43 \%$ at long-term intervals.

Bruscia (1991) describes a case study which illustrates the effect of music on a severely regressed Alzheimer's sufferer $(\mathrm{Mr} \mathrm{O})$ of 66 years of age. The study describes 15 months of weekly group music therapy which show that the patient's behaviour did not change significantly over an 11-week period despite deterioration in physical and cognitive capacities over the duration of the study. At this stage, $\mathrm{Mr} \mathrm{O}$ was referred to the music therapist as he no longer had the cognitive ability to engage in other activities. $\mathrm{MrO}$ was generally unresponsive, agitated and restrained in a 'Ger-Chair'. Weekly group sessions lasting $30 \mathrm{~min}$ involved singing songs and imitating rhythms. $\mathrm{Mr} \mathrm{O}$, who did not usually speak, participated by singing whole songs and as his condition declined, choruses only. Mr O's eye contact with the therapist and other members of the group was intense compared with his unresponsiveness at most other times. His rhythm was consistently appropriate and his concentration on other group members as they played was continuous. As his condition worsened he still participated at his maximum level by turning his head towards the sound, thus still responding to auditory stimulation. Bruscia also points out that, as with $\mathrm{MrO}$ and many other patients, music is the only other stimulation besides food that attracts attention. As the disease progressed, $\mathrm{Mr} \mathrm{O}$ became less functional and more isolated. However, he and other patients still had sensory and emotional requirements. Bruscia (1991) says that music can meet these requirements through group interaction to bring the regressed patient out of isolation. Bruscia is not alone in this belief in that many care givers skilled in the use of music therapy have reported increased group cohesion, stimulation of memory and expression of emotion, and greater rhythmic movement and touching behaviours resulting from music therapy (Palmer, 1983; Bailey, 1985; Bright, 1990). However, there has been little empirical evidence to substantiate these reports.

Glynn (1992) also claims that music can be used as a therapeutic modality to help Alzheimer's patients achieve the highest possible level of functioning. He also highlights 
the lack of empirical research available to evaluate the immediate and sustained physiological, psychological and psycho-social therapeutic effects of music therapy on behavioural patterns of institutionalized Alzheimer's sufferers. Glynn (1992) believes that, because of the debilitating nature of the disease both physically and psychologically, functional effectiveness and human freedom and dignity must be maximized. He also highlights the lack of research aimed at defining interventions to maximize psycho-social function.

Palmer (1983) also suggests that music can motivate and stimulate behavioural repatterning and many elderly people will sway rhythmically, tap their feet, experience an increased motivation to mobilize the upper extremities and expand their limited range of movement. The psychological results have been described as enhanced self-esteem, reality orientation and increased social awareness. However, no tool has been developed to quantify the interplay of the biopsychosocial dimensions of Alzheimer's patients with their environment. It was for this reason that the Music Therapy Assessment Tool (MTAT) was developed to measure the effects of music on the behavioural patterns of Alzheimer's sufferers within a given environment by Glynn (1992). The pupose of Glynn's study (1992), which involved 20 patients, was to test the MTAT for inter-rater reliability and internal consistency. The results showed that the MTAT has a 'fairly high' inter-rater reliability and internal consistency, thus establishing it as a viable tool for use in further research into music therapy.

\section{Parkinson's disease and music therapy}

It is not only for Alzheimer's disease that robust evidence for the use of music therapy as a viable therapeutic option is lacking. Again for Parkinson's disease, there are many positive accounts of the success of music therapy in improving quality of life for patients by maximizing social, physical and psychological functioning, but scant empirical evidence to reinforce these valid yet subjective accounts. Swallow (1987) describes a project developed in Claremont Hospital, Belfast, which looked at ways in which techniques in music therapy could be applied to Parkinson's disease. The basis for this project was the premise that music could be used in conjunction with physiotherapy and the suggestion that music could be used to overcome the characteristic frustrations of variations in motor function. Swallow (1987) argues that music therapy techniques can be used to regularize walking patterns, prevent akinetic freezing, improve posture and control of upper limb movement and promote relaxation. Swallow's study involved 11 patients with varying levels of disability with whom
2-3 h each morning were spent in groups or individually with a physiotherapist and two music therapists. Simple exercises were designed to overcome particular problems, with the accompaniment of improvised piano, flute or guitar music. To improve walking or balancing the patient's own natural walking rhythm was matched by the instrument. This resulted in lengthening and regularizing of the stride and decreases or increases in speed as the tempo of the music changed. Such variation in walking for sufferers of Parkinsonism is difficult and was therefore quite an achievement. Stopping and starting with the music could also be practised. Musical exercises to improve posture involved rhythmic swaying and torsional movements. Simple repeated musical phrases can reinforce swaying movements which must precede rising from a chair or turning in bed.

Swallow (1987) also suggested that a combination of music therapy and speech therapy could improve speech. During another phase of the project, chanting and singing were used to improve articulation and Kazoos (small, cigar shaped instruments through which air is blown to create a nasal, buzzing sound) were used to improve breathing and breath control. In Parkinson's disease, dyskinetic movements are worsened by embarrassment or anxiety. Music was used to affect mood and promote relaxation, thus reducing tremor and rigidity which, in turn, improved voluntary movement. Unfortunately, as Swallow acknowledges, there has been no objective assessment of this work. However, he indicates that patients and carers responded by stating that an increase in physical activity and reduction in fatigue was brought about by the use of music.

Swallow (1987) questions how music actually works and how much benefit is due to the music or to the rest of the therapy. The author places great importance on group activities, observing the way in which music helped to break down the barriers created by disability and selfconsciousness, drawing people together. Lastly Swallow makes reference to the carry-over effect of music therapy in this context, stating that patients experienced an improvement in movement for only a short period of time $(24 \mathrm{~h})$ after therapy. In some cases, however, it was possible to prolong the benefit by consciously recalling musical associations. This study, although positive in its claims of the effects of music therapy, draws only on the experiences of 11 patients and is limited by a lack of objective evaluation.

Selman (1988) provides another useful case study regarding Parkinson's disease and music therapy. His paper, however, focuses not on physical disabilities of the disease, but on psychological implications of the accompanying communication deficits. In this case music is used as a communicative channel through which feelings of frus- 
tration and anger can be vented when this is not possible with often dysarthric and incomprehensible speech. Selman uses the example of a patient called John, for whom the aim of music therapy was to facilitate expression, allowing emotional relief. As his speech and gestures were poor due to lack of muscular control, and facial expression almost impossible, music seemed the best communicative medium. Music therapy was used in conjunction with physiotherapy and relaxation therapy. Singing lessons were initiated as a physical exercise to release the jaw and tongue. As the lessons progressed, a decline of rigidity occurred and work on posture and breathing out were begun. Later sessions involved singing prescribed words in short turntaking phrases. The author describes the intimate nature of lessons and the encouragement required to overcome John's reluctance to use his voice. Improvizations using drum and xylophone, although exhibiting the festination and tremor characteristics of Parkinson's, did not exhibit the negative psychological effects of the disease such as impoverishment of feeling, motive or intention. Selman (1988) further describes John's music as 'determined' and 'free' from the bonds of illness. Physical and emotional release was achieved through outbursts of music-making, with the positive effects of this lasting up to $24 \mathrm{~h}$. Though this article does not provide empirical evidence to support the use of music therapy in Parkinson's disease, it does provide a useful description of successful music therapy with one patient and thus, potentially, many more.

\section{Pain relief and music therapy}

The use of music therapy in the relief of pain is relevant to care of the elderly and therefore merits brief mention. Many elderly people suffer chronic pain resulting from arthritis or cancer for example. Schorr (1993) investigated the use of music as a unitary-transformative means of altering the perception of pain amongst women with rheumatoid arthritis. In a repeated measures investigation, 31 women with rheumatoid arthritis responded to the McGill Pain Questionnaire (Melzack, 1975) prior to listening to music of their choice, during music and 1-2 $\mathrm{h}$ after the intervention. Analysis of the data supported the use of music as a means of lessening pain perception. Results showed that pain thresholds increased while subjects listened to music for a period of time following the intervention. Beck's (1991) study also supports the theory of music as a pain reduction tool. Beck assesses the extent to which the therapeutic use of music would reduce pain in cancer patients. The experiment involved 15 patients who were assigned randomly to listen either to music of their preference or to a control intervention in the form of a 60 cycle hum. The results showed a statistically significant decrease in pain from using either the music or the sound, though the mean percentage of change in pain for music was twice that for sound. These findings support the use of music as an independent nursing intervention to relieve pain. Rozanno \& Locsin's (1981) study also provided positive results with the use of music in inducing pain relief. They found that when music was used with postoperative patients, they had fewer overt pain reactions and required less medication than patients in the no music group.

From these three studies, it seems that the use of music to relieve pain is valuable. Music intervention could be applied to pain relief in the elderly although more research is necessary to assess its use in this specific area. The risks of using music in pain relief are minimal, the costs low and the potential to reduce suffering high.

Also of note is the use of music to aid relaxation and reduce sleep disturbance in elderly care wards. Strang (1970) carried out a study involving 32 patients. Each evening for 3 months music was played after the patients went to bed. This resulted in an increase from eight to 27 in the number of patients sleeping well without night sedation.

\section{Conclusion}

From this review of literature, it seems that music therapy indeed has potential as a useful therapeutic intervention with a wide variety of client populations through a variety of mechanisms. Music is said to be unique in that it can penetrate both the mind and the body directly, whatever the individual's intelligence or condition. Music acts to stimulate the senses, evoking feelings and emotions, it causes physiological and mental responses and energises the body and mind. It seems that music can be successful in reducing the social, emotional, communicative and physical disabilities incurred by dementia, Alzheimer's or Parkinson's disease. However, it is also recognized that more research is required to measure the actual effects and benefits of music therapy in specific conditions. It is important to establish whether there is a causal link between music therapy and outcome. This is especially pertinent in the present harsh economic climate where increasing demand is made on an NHS with limited resources. To justify an expansion in the use of music as a therapeutic modality, there must be more compelling evidence to validate music therapy's efficacy (Radhakishnan, 1991).

\section{References}

Bailey L.M. (1985) Music's soothing charms. American Fournal of Nursing 85(11), 1280. 
Beck S. (1991) The therapeutic uses for cancer related pain. Oncology Nursing Forum 18(8), 1327-1337.

Bruscia K. (1991) Case Studies in Music Therapy. Barcelona Publishers, Phoenixville, PA, p. 573.

Crystal H., Grober E. \& Mauser D. (1989) Preservation of musical memory in Alzheimer's disease. Fournal of Neurology, Neurosurgery and Psychiatry 52, 1415-1416.

Evans M. \& Rubio P. (1994) Music: a diversionary therapy. Today's OR Nurse 16(4), 17-22.

Fitzsimmons L. (1991) Variables influencing cardiovascular function. Fournal of Cardiovascular Nursing 5(4), 87-89.

Gerdner L. \& Swanson E. (1993) Effects of individualised music on confused and agitated elderly. Archives of Psychiatric Nursing 7(5), 284-291.

Glynn N.J. (1992) The music therapy assessment tool in Alzheimer's patients. Fournal of Gerontological Nursing 18(1), 5-9.

Kawano T. (1992) Music therapy: application for psychosomatic disease in senescence. Fapanese Fournal of Psychosomatic Medicine 32, 115-120 (abstract).

Melzack R. (1975) The McGill Pain Questionaire: major properties and scoring methods. Pain 1, 277-299.
Norberg A. (1986) Reactions to touch, music and object presentation in the final stages of dementia: an exploratory study. International Fournal of Nursing Studies 23(4), 315-323.

Palmer M. (1983) Alzheimer's and critical care. Fournal of Gerontological Nursing 9, 86-90.

Pavlicevic M.C. (1991) Music therapy in Scotland; an introduction. Health Bulletin 49(3), 91-94.

Radhakishnan G. (1991) Music therapy: a review. Health Bulletin 49(3), 195-200.

Rozanno G. \& Locsin R.J. (1981) The effect of music on the pain of selected post-operative patients. Fournal of Advanced Nursing 6, $19-25$.

Schorr A. (1993) Music and pattern change in chronic pain. Advances in Nursing Science 15(4), 27-36.

Selman J. (1988) Music therapy with Parkinson's. British Fournal of Music Therapy, part 2, 5-9.

Strang C.D. (1970) Music at night: an experiment in a geriatric ward. Fournal of the Royal College of Practitioners 20(99), 246-247.

Swallow M. (1987) Can music help? Current Problems in Neurology 6, 109-112. 